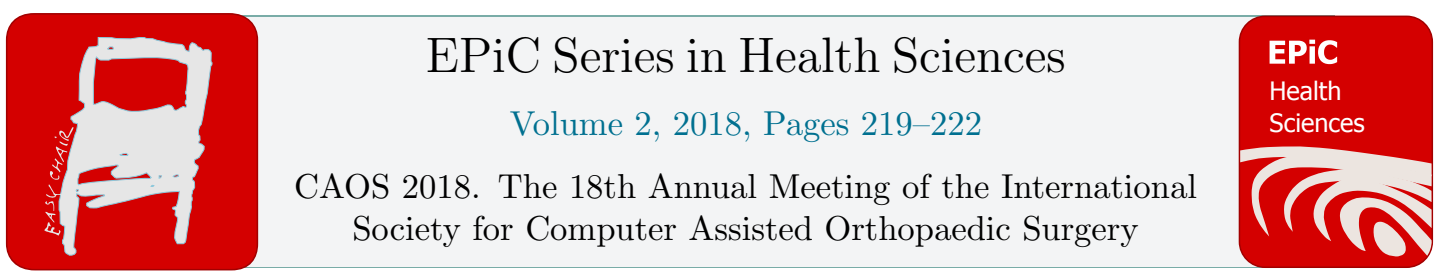

\title{
Why Did Anterior Dislocation Occur during Dandling Her Baby in front of Her in Standing Position after THA?
}

\author{
Kunihiko Tokunaga ${ }^{1}$, Juliana $\mathrm{Hsu}^{2}$, Maximilian C. M. Fischer ${ }^{2}$, Masashi \\ Okamoto $^{3}$, and Klaus Radermacher ${ }^{2}$ \\ ${ }^{1}$ Niigata Hip Joint Center, Kameda Daiichi Hospital, Niigata City, 950-0165, Japan \\ ${ }^{2}$ Chair of Medical Engineering, Helmholtz Institute for Biomedical Engineering, RWTH Aachen \\ University, Germany \\ ${ }^{3}$ Department of Radiology, Kameda Daiichi Hospital, Niigata City, 950-0165, Japan \\ ktokunagajp@yahoo.co.jp, jhsu@hia.rwth-aachen.de
}

\begin{abstract}
We experienced an anterior dislocation of a lady after THA in standing position during dandling her baby in front of her. The orientations of acetabular cup and femoral stem, measured by 3D image analysis and post-operative CT images, were within the so called safe zone. As it happened, the moment of this dislocation was captured in a smart phone movie. Surface 3D models were created from pre- and post-operative CT images. The pelvic sagittal tilt angle was measured using preoperative EOS image in standing position and landmark-based 2D/3D registration. The pelvic tilt and the post-operative THA parameters served as input to calculate the cup orientation relative to the prosthetic ROM-based target zone, the bone stock-based target zone, and maximal amount of external rotation until "bony impingement". The prosthetic ROM-based target zone algorithm calculates impingement free cup orientations dependent on the stem orientation, neck shaft angle and pelvic orientation. The system clearly showed that impingements occurred during the slight hip external rotation and leaning back motions of the patient in standing position as shown in the smart phone movie. This system demonstrated the neck shaft angle of the stem should be reduced from $135^{\circ}$ to $130^{\circ}$, and the stem antetorsion should be reduced from $35^{\circ}$ to $20^{\circ}$ to get optimum target zone without impingements. In addition, the cup should be placed at $6 \mathrm{~mm}$ posteriorly to get maximum bony coverage. This patient-specific target zone calculation is a powerful tool to decide the optimum orientation and position of THA implants.
\end{abstract}




\section{Introduction}

The patient (38 years old female) underwent right total hip arthroplasty (THA) using a CT-based hip navigation system. She dislocated her hip joint during dandling her baby in front of her in standing position at 3 months after THA. The smart phone movie of her mother captured the moment of this dislocation. She held her baby up in her arms by tilting her back posteriorly with slight external rotation of her left hip joint. Suddenly she lost her balance and fell down on grass ground.

The X-ray films showed anterior dislocation of her hip joint which was reduced under spinal anesthesia. A hip abduction brace was applied for 3 months and she never hold her baby in front of her. Fortunately dislocation never happened for over 3 years after that event. The implant positions measured using postoperative CT images and a 3D analyzing software demonstrated that implant positions were within a so called safe zone [Lewinnek et al. 1978], [Sugano et al. 2012]. A tool for patient-specific target zone calculation was used to reveal the reason of this anterior dislocation.

\section{Material and Methods}

The pre-operative pelvic tilt in standing position was measured using landmark-based 2D/3D registration of the pelvis model and the pre-operative EOS images (EOS imaging Inc. Paris, France). Pre- and post-operative 3D surface models were created from the CT images. The post-operative surface model, including bony and implant surfaces, was registered to the pre-operative surface models. The orientation and position of the cup and stem relative to the anterior pelvic plane for pelvic and table top plane for femoral coordinates were measured within the surface models.

The pelvic tilt and the post-operative THA parameters served as input to calculate the cup orientation relative to the prosthetic ROM-based target zone, the bone stock-based target zone, and maximal amount of external rotation until "bony impingement". The prosthetic ROM-based target zone algorithm calculates impingement free cup orientations dependent on the stem orientation, neck shaft angle and pelvic orientation [Hsu et al. 2017]. The ROM defined by Yoshimine et al. [Yoshimine 2006] was used as the desired ROM. The actual implanted cup orientation is compared to the resulting ROMbased target zone. Possible modifications of the stem parameters were also investigated to determine whether a different stem combined with the actual cup orientation would yield an impingement free result. For the bone-stock-based target zone, the bony coverage of the cup were calculated for the postoperative situation. Variations of the cup positions were also investigated in order to achieve higher cup coverage. For calculating the maximum external rotation (ER), three different values for pelvic tilt were used: the measured pelvic tilt in the pre-operative EOS imaging $\mathrm{t}, \mathrm{t}-5^{\circ}$ and $\mathrm{t}-10^{\circ}$. The latter values were assumed to better approximate the posture of the patient (carrying her baby in the front) when the dislocation happened.

\section{Results}

The measured THA parameters from CT data are as follows: radiographic inclination: $44^{\circ}$, radiographic anteversion: $19^{\circ}$, cup position relative to pre-operative acetabular centre: $6 \mathrm{~mm}$ cranial, $13 \mathrm{~mm}$ medial and $0 \mathrm{~mm}$ anterior/posterior, stem antetorsion: $35^{\circ}$, stem adduction: $7^{\circ}$, stem flexion: $3^{\circ}$, stem position: $15 \mathrm{~mm}$ cranial, $14 \mathrm{~mm}$ medial, and $10 \mathrm{~mm}$ anterior. The pre-operative pelvic tilt in standing position is $-11^{\circ}$ (posterior tilt). Using the post-operative THA parameters, bony impingement occurred at $<20^{\circ}$ ER for $-11^{\circ}$ pelvic tilt, $<15^{\circ}$ ER for $-16^{\circ}$ pelvic tilt and $<10^{\circ}$ ER for $-21^{\circ}$ pelvic tilt. The prosthetic ROM-based target zone analysis showed that the actual cup orientation is outside of the 
target zone. If we set the cup orientation inside the new target zone: neck shaft angle from $135^{\circ}$ to $130^{\circ}$, and stem antetorsion from $35^{\circ}$ to $20^{\circ}$, the maximum amount of ER for $-21^{\circ}$ pelvic tilt became $>20^{\circ}$. In addition, the bone-stock-based target zone calculation showed that when changing the cup position by $6 \mathrm{~mm}$ posteriorly, the cup coverage increased from $76 \%$ to $89 \%$.

\section{Discussion}

Even though her acetabular cup and femoral stem were placed within a so called safe zone [Lewinnek et al. 1978] [Sugano et al. 2012], anterior dislocation occurred in standing position during dandling her baby in front of her. The tool for patient-specific target zone calculation [Hsu et al. 2017] clearly explained the impingements during the slight ER motion of the patient as shown in the smart phone movie. Hip surgeons usually decide acetabular cup orientation according to the pelvic coordinates in supine position, such as APP or functional pelvic plane coordinates [Nishihara et al. 2003]. The result of this study demonstrated hip surgeons had to consider pelvic sagittal tilt in standing position to decide the implant orientation. However, it is difficult to estimate how much changes occur in pelvic sagittal tilt after THA and we have to solve this issue. The patient-specific target zone calculation also provide to use stem with less than $135^{\circ}$ of neck shaft angle as shown by Widmer [Widmer et al. 2005]. The stem antetorsion angle also plays very important role to avoid bony or implant impingements. To control the orientations of acetabular cups and femoral stems, we may need to use precise navigation or robotic systems for THA. This patient-specific target zone calculation seems to be a powerful tool to decide the optimum orientation of THA implants. We need to evaluate this system with large number of cases.

\section{References}

[Lewinnek et al. 1978] LEWINNEK GE, Lewis JL, Tarr R, Compere CL, Zimmerman JR Dislocations after total hip-replacement arthroplasties. J Bone Joint Surg Am. 60(2) Lewinnek GE, Lewis JL, Tarr R, Compere CL, Zimmerman JR Dislocations after total hip-replacement arthroplasties. J Bone Joint Surg Am. 60(2) (1978):217-220

[Sugano et al. 2012] SUGANO N, Tsuda K, Miki H, Takao M, Suzuki N, Nakamura N Dynamic measurements of hip movement in deep bending activities after total hip arthroplasty using a 4dimensional motion analysis system. J Arthroplasty 27(8):(2012) 1562-1568

[Hsu et al. 2017] Hsu, J; DE LA Fuente, M; RADERMACHER, K.: Multi-dimensional Range-of Motionbased safe zone for patient-specific total hip arthroplasty. In: 17th Annual Meeting of the International Society for Computer Assisted Orthopaedic Surgery (2017)

[Yoshimine 2006] Yoshimine, F.: The safe-zones for combined cup and neck anteversions that fulfill the essential range of motion and their optimum combination in total hip replacements. In: Journal of biomechanics, 39 (2006), 7: 1315-1323

[Nishihara et al. 2003] NISHIHARA S, Sugano N, Nishii T, Ohzono K, Yoshikawa H. Measurements of pelvic flexion angle using three-dimensional computed tomography. Clin Orthop Relat Res. 411:(2003) 140-51.

[Widmer et al. 2005] WIDMER KH, Majewsk, IM. The impact of the CCD-angle on range of motion and cup positioning in total hip arthroplasty. Clin Biomech 20(7): (2005)723-728 


\section{Disclosures}

The authors declare that they have no conflict of interest. 